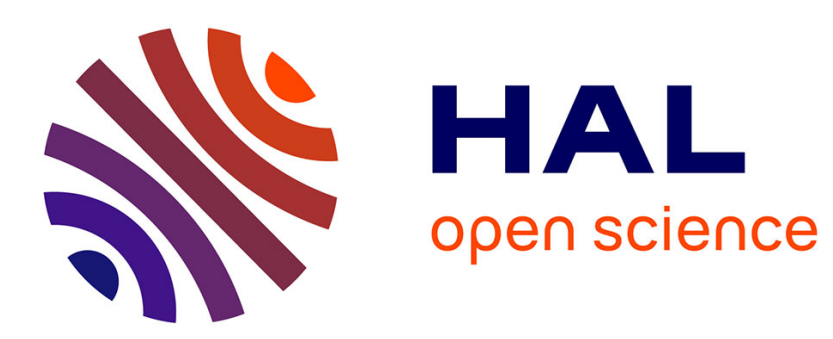

\title{
Energy-based stability analysis for grasp selection with compliant multi-fingered hands
}

\author{
Ugo Vollhardt, Maria Makarov, Alex Caldas, Mathieu Grossard, Pedro \\ Rodriguez-Ayerbe
}

\section{- To cite this version:}

Ugo Vollhardt, Maria Makarov, Alex Caldas, Mathieu Grossard, Pedro Rodriguez-Ayerbe. Energybased stability analysis for grasp selection with compliant multi-fingered hands. ECC 2019 - European Control Conference, Jun 2019, Naples, Italy. pp.1592-1597, 10.23919/ECC.2019.8795792 . hal-02499342

\section{HAL Id: hal-02499342 \\ https://hal.science/hal-02499342}

Submitted on 5 Mar 2020

HAL is a multi-disciplinary open access archive for the deposit and dissemination of scientific research documents, whether they are published or not. The documents may come from teaching and research institutions in France or abroad, or from public or private research centers.
L'archive ouverte pluridisciplinaire HAL, est destinée au dépôt et à la diffusion de documents scientifiques de niveau recherche, publiés ou non, émanant des établissements d'enseignement et de recherche français ou étrangers, des laboratoires publics ou privés. 


\title{
Energy-based stability analysis for grasp selection with compliant multi-fingered hands
}

\author{
Ugo Vollhardt ${ }^{1,2}$, Maria Makarov ${ }^{2}$, Alex Caldas ${ }^{3}$, Mathieu Grossard ${ }^{1}$, Pedro Rodriguez-Ayerbe ${ }^{2}$
}

\begin{abstract}
This article presents a stability analysis of object grasping with a compliant multi-fingered robot hand. This stability analysis is based on an energetic approach, in which we aim to compute the maximum amount of energy that can be stored by a compliant hand-object system about a stable equilibrium before being destabilized. More specifically, the case of external disturbance wrenches is investigated. This analysis admits several applications, two of which are illustrated in this article on a case study. First the quality of a given grasp can be quantified in terms of maximum allowed disturbance force and the associated displacement around the initial equilibrium, with applications in trajectory design for the robot arm that holds the hand-object system. Conversely, given a task specified in terms of known disturbance wrenches set, the proposed approach helps determining the optimal grasp for an enhanced stability, e.g. the contact points locations, joint compliance or internal forces levels.
\end{abstract}

\section{INTRODUCTION}

Grasping and manipulating objects with complex grippers, such as multi-fingered hands is an arduous and challenging task in robotics and is still an active field of research [1]. The goal of a successful grasp is to maintain an object in the hand under external perturbation without damaging it. The evaluation of the quality of the grasp can help to find the best grasping parameters according to some criteria (robustness, task dependence, dexterous behavior, etc.).

A sub-category of the multi-fingered hands are the compliant hands, in which compliant elements are located on the fingertips, in the finger segments or within the joints, being in this latter case either passive or active through joint controller gains. These type of hands are conveniently described by object-level stiffness models and energetic approach for their control.

The stability of a grasp is one of the most important aspects of the grasp quality [2] and it is defined for a grasp at an equilibrium. An equilibrium for a grasp corresponds to the situation when the sum of all the forces and moment acting on the grasped object equals zero. When all forces and moments are derived from a potential function, an equilibrium is said stable if the first-order derivative of the

\footnotetext{
${ }^{1} \mathrm{U}$. Vollhardt and M. Grossard are with the Interactive Robotics Laboratory of CEA-LIST 91192 Gif sur Yvette Cedex, France, ugo.vollhardtecea.fr, mathieu.grossardecea.fr

${ }^{2}$ U. Vollhardt, M. Makarov and P. Rodriguez-Ayerbe are with the Signals and Systems Laboratory of CentraleSupélec, CNRS, University Paris-Sud, University Paris-Saclay, 91192 Gif sur Yvette Cedex, France, maria.makarov@centralesupelec.fr, pedro.rodriguez@centralesupelec.fr

${ }^{3}$ A. Caldas is with the ESME Research Lab, ESME Sudria Engineering school, 38 Rue Molière, 94200 Ivry-sur-Seine, France, alex.caldascesme.fr
}

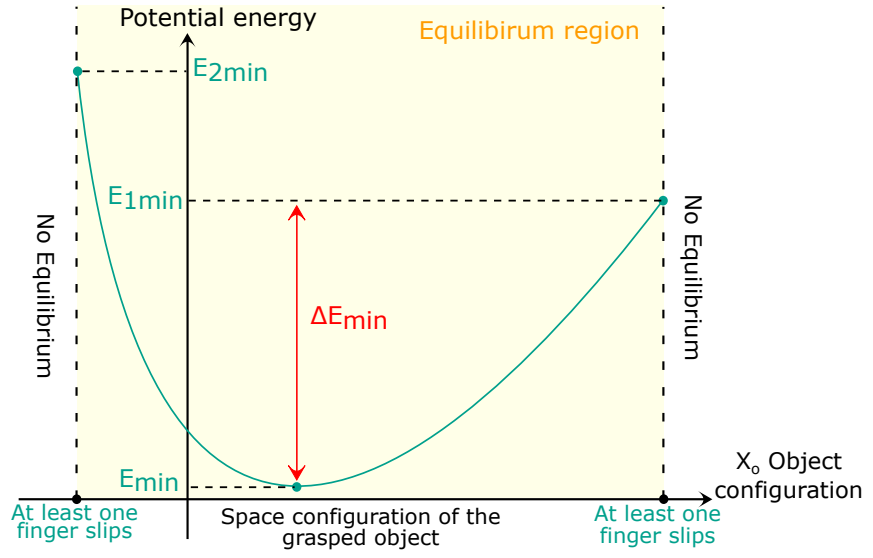

Fig. 1: Stable equilibrium region described using the grasp potential energy (based on [8]), concept illustration in 1D

potential energy is null and the matrix of second-order partial derivatives (corresponding to stiffness matrix) is positive definite [3].

In this paper, a characterization of the stability of an equilibrium grasp is proposed for compliant multi-fingered hands. the proposed criterion takes into account the whole system composed of the controlled hand and the object, which allows to evaluate the influence of multiple parameters such as the joint servo gains or the contact points choices.

One classical way to ensure the grasp stability is by inspecting the force-closure property [4], which can be often encountered in the dedicated literature because this property implies a stable equilibrium grasp [5]. Although this is a useful property, it can be a conservative one since it is possible to achieve stability without force-closure [6]. The stability of a system that is not force-closure is influenced by three factors, namely the contact points, the compliance at the contact points and the local curvature at the contact points.

Specifically dealing with the second point, several studies conducted a stability analysis based on the grasp stiffness definition and its properties analysis. Bruyninckx et al. proposed several indexes of stability based mainly on the eigenvalues of the stiffness matrix [2]. Howard and Kumar [6] also produced a stability criterion based on the stiffness, as well as a method to compute the related stiffness matrix based on the local curvature at the contact point. Cutkosky et al. [7] introduced another index that takes into account the structural compliance of the hand-object system, as well as the compliance contributed by the servoing of the joints.

The study of the stiffness for the grasp stability also led to energetic approaches, as in Tsuji et al. [8]. In their 
work, Tsuji et al. proposed a stability index expressed as the minimal amount of energy needed to destabilize a grasp (see Fig. 1).

This paper presents a grasp stability analysis based on a energetic approach. This approach takes in consideration the elastic deformation of the fingertips in the same way as proposed by Tsuji et al. [8], but But, our approach generalizes it in integrating the whole geometry of the hand as well as the stiffness brought by the joints into the system. This approach is well suited for compliant hands, with passive or active elastic joints. This analysis is task-oriented, and allows to select and tune a grasp specialized for a task to guarantee it's stability to the class and scale of external disturbances specific to the task.

Section II exposes the grasp quality measure using the energetic approach. The analysis of the grasp stability is proposed in Section III. Section IV is dedicated to an illustrative case study, and conclusions are given in Section V.

\section{ENERGETIC APPROACH}

This section provides a brief overview of the framework proposed by Tsuji et Al. [8], and details our contribution which allows to evaluate the stability region around the equilibrium when the system is under an external disturbance.

\section{A. Minimum destabilizing energy}

The index of grasp stability developed by Tsuji et al. [8] is based on the principle that, as the object is grasped using elastic fingertips, it is possible to construct a potential field around the stable equilibrium point. This potential field can be divided in two parts, the region around the stable equilibrium point where no fingertip slips, denoted as the "equilibrium region" and the region around this equilibrium region where at least one fingertip slips (see Fig. 1).

The equilibrium region has a point where the potential energy is minimal, the energy at this point is denoted $E_{\text {min }}$. In addition, each boundary of the region (which should be seen as multidimensional whereas the Fig. 1 only conveys the concept in one dimension) also has a minimum, denoted respectively $E_{1_{\text {min }}}, \ldots, E_{n_{m i n}}, n$ being the number of boundaries.

Thus, the index of grasp stability proposed [8], denoted as $\Delta E_{m i n}$, is the minimum difference between $E_{\text {min }}$ and $E_{1_{\text {min }}}, \ldots, E_{n_{\text {min }}}$, as follows:

$$
\Delta E_{\text {min }}=\min _{i=1, \ldots, n}\left\{E_{i \min }-E_{\text {min }}\right\}
$$

\section{B. Direction dependent minimum destabilizing energy and wrench}

Although the previously described approach provides an interesting index, it is a general-purpose index that gives the minimal amount of energy required to destabilize the grasp regardless of the direction of the perturbation that supplies this energy. Moreover, only the fingertips and not the hand were considered to compute the stiffness.

Based on the previous approach, instead of considering the minimal energy of the boundaries of the equilibrium region and thus exploring all the directions of this space, we consider only a specific set of directions which is linked to a specific set of external wrenches. This proposition allows to evaluate the robustness of the grasp to external disturbances in specific directions, e.g. according to the manipulation task. Moreover this point is found using the whole kinematic of the hand and not just the fingertips as described in the following subsections.

\section{Computation of the minimum destabilizing energy and wrench}

In a 3D grasp case (respectively 2D grasp case), a contact $c_{i}$ between a fingertip $i$ and an object can be described as a mapping between the forces $f_{c_{i}}$ applied by the finger on the contact point in the contact frame $C_{i}$, and the resulting wrench $W_{d p \rightarrow o b j}^{o} \in \mathbb{R}^{6}$ (resp. $W_{d p \rightarrow o b j}^{o} \in \mathbb{R}^{3}$ ) to which the object is submitted in its own coordinate frame obj (denoted by the superscript $\bullet^{\circ}$ ).

The concatenation of all the contact forces $f_{c}=$ $\left[f_{c_{1}}^{T} f_{c_{2}}^{T} \ldots f_{c_{n}}^{T}\right]^{T}, n$ being the number of contact points between the object and the fingers, and the resulting wrench applied on the object $W_{d p \rightarrow o b j}^{o}$ are related by:

$$
W_{d p \rightarrow o b j}^{o}=G f_{c}
$$

where $G \in \mathbb{R}^{6 \times n}$ is the grasp map built from the adjoint matrices relating the contact frames to the object frame and depends on the contact type. In this article, the Point Contact with Friction (PCwF) model is used [4].

The contact forces $f_{c}$ can be split in two components. The forces that induce a motion of the object are denoted as reaction forces $f_{r}$ and the forces that do not induce a motion of the object are denoted as internal forces $f_{i}$. The forces $f_{i}$ belong to the null space of $G$.

$$
f_{c}=f_{r}+f_{i}, f_{i} \in \operatorname{Null}(G)
$$

Let us now consider an external wrench applied on the object in the world frame $w$ (denoted by the superscript $\left.\bullet^{w}\right), W_{e x t \rightarrow o b j}^{w}=\alpha d_{W_{e x t}}, d_{W_{e x t}} \in \mathbb{R}^{6}$ being its direction and $\alpha \in \mathbb{R}$ its intensity, $\alpha_{\max }$ the maximum intensity of $W_{e x t \rightarrow o b j}^{w}$ for which no slippage of contact point occurs.

For any $\alpha \in\left[0, \alpha_{\max }\right]$, if the system is at an equilibrium, it means the wrench applied on the object by the fingers in $w, W_{d p \rightarrow o b j}^{w}$ is related to $W_{e x t \rightarrow o b j}^{w}$ by:

$$
W_{e x t \rightarrow o b j}^{w}=-W_{d p \rightarrow o b j}^{w}
$$

Let us denote $E_{d_{w_{e x t}} \max }$, the energy stored in the grasp when the system is at the equilibrium point corresponding to the external wrench $W_{e x t \rightarrow o b j}^{w}=\alpha_{\max } d_{W_{e x t}}$, then:

$$
\Delta E_{\min }=E_{d_{w_{e x t}} \max }-E_{\min }
$$

In the rest of this article, we consider that the potential energy of the grasp is null when the grasp is not under a disturbance wrench. Thus $E_{\min }$ is null, as it is only an offset determined by the initial configuration of the grasp. 


\section{Calculation of Potential Energy}

For a given contact point $i$ between the object and a fingertip, let us denote $d_{n_{i}}, d_{s_{i}} \in \mathbb{R}^{3}$, the deformation of the fingertip and $f_{n_{i}}, f_{s_{i}} \in \mathbb{R}^{3}$ the contact forces, in normal direction of the contact surface and tangential direction respectively. Assuming that the relationship between the deformation and contact forces is expressed by a linear spring model, the forces are defined as follows:

$$
f_{n_{i}}=-k_{n} d_{n_{i}}, \quad f_{s_{i}}=-k_{s} d_{s_{i}}
$$

with $k_{n}$ and $k_{s} \in \mathbb{R}$ being respectively the stiffness in the normal and shear direction of the contact surface. Moreover let the displacement of the $i$-th contact point be $\Delta X_{c_{i}}=$ $\left[\Delta X_{c_{i_{x}}}, \Delta X_{c_{i_{y}}}, \Delta X_{c_{i_{z}}}\right]^{T}$. According to [8], the variation of potential energy is given by:

$$
\Delta E=\frac{1}{2} \sum_{i=1}^{n}\left\{k_{n} \Delta X_{c_{i_{z}}}^{2}+k_{s}\left(\Delta X_{c_{i_{x}}}^{2}+\Delta X_{c_{i_{y}}}^{2}\right)\right\}
$$

Let $k_{c_{i}}=\operatorname{diag}\left(k_{s}, k_{s}, k_{n}\right)$, so (7) becomes :

$$
\Delta E=\frac{1}{2} \sum_{i=1}^{n} \Delta X_{c_{i}}^{T} k_{c_{i}} \Delta X_{c_{i}}
$$

Then, if $K_{c}$ denotes the matrix containing on its diagonal the matrices $k_{c_{i}}$ and $X_{c}$ the concatenation of the coordinates of the contact points $X_{c_{i}}$ in the object frame obj, (7) becomes:

$$
\Delta E=\frac{1}{2} \Delta X_{c}^{T} K_{c} \Delta X_{c}
$$

\section{E. Grasp Stiffness}

The stiffness of a grasp can be defined as the relationship between a external wrench applied on the grasped object and its resulting motion [7]. If the configuration of the object in the world coordinate frame $w$ is denoted $X_{o}^{w} \in \mathbb{R}^{6}$, the stiffness seen by the object in $w$ is:

$$
K_{o}^{w}=\frac{\partial W_{e x t \rightarrow o b j}^{w}}{\partial X_{o}^{w}}=-\frac{\partial W_{d p \rightarrow o b j}^{w}}{\partial X_{o}^{w}}
$$

This stiffness can also be similarly expressed in the $o b j$ frame

$$
K_{o}^{o}=-\frac{\partial W_{d p \rightarrow o b j}^{o}}{\partial X_{o}^{o}}
$$

$\delta W_{d p \rightarrow o b j}^{o}$ and $\delta X_{o}^{o}$ being respectively a small variation of the wrench applied by the fingers on the object and a small displacement of the object, in the $o b j$ frame. By the chain rule and using (2), we have:

$$
K_{o}^{o}=-\underbrace{G \frac{\partial f_{c}}{\partial X_{o}^{o}}}_{K_{b}}-\underbrace{\frac{\partial G}{\partial X_{o}^{o}} f_{c}}_{K_{j}}
$$

$K_{b}$ is the contribution of the structure and servoing of the joint to the stiffness of the hand, whereas $K_{j}$ is the contribution induced by the variations of the grasp while the object is moving. In this article the contact points are considered to be fixed so that $K_{j}$ is null in this approximation and $K_{o}^{o}=K_{b}$. The relationship between a variation of the wrench $\delta W_{d p \rightarrow o b j}^{o}$ and a small motion $\delta X_{o}^{o}$ of the object is:

$$
\delta W_{d p \rightarrow o b j}^{o}=-K_{o}^{o} \delta X_{o}^{o}
$$

Using the grasp map $G$, it is possible to express the stiffness in the frames of the contact points. The latter can be convenient as the friction cone constraints are often checked in these frame in the rest of this paper. Let us denote $K_{c}$ the stiffness matrix in the contact frame, that relates a small motion of the contact point $\delta X_{c}$ to a variation of contact forces $f_{c}$ :

$$
\delta f_{c}=K_{c} \delta X_{c}
$$

Using the grasp map, it is possible to express the small motion of the contact points $\delta X_{c}$ out of a small motion of the object $\delta X_{o}^{o}$ :

$$
\delta X_{c}=G^{T} \delta X_{o}^{o}
$$

Using (2), (14) and (15), the expression of $W_{d p \rightarrow o b j}^{o}$ is:

$$
\delta W_{d p \rightarrow o b j}^{o}=-\underbrace{G K_{c} G^{T}}_{K_{o}^{o}} \delta X_{o}^{o}
$$

As described in [9], the stiffness $K_{c}$ can be defined by:

$$
K_{c}=\left(C_{s}+J_{h}(q) C_{q} J_{h}(q)^{T}\right)^{-1},
$$

where $C_{s} \in \mathbb{R}^{3 n \times 3 n}$ (resp. $\mathbb{R}^{2 n \times 2 n}$ for $2 \mathrm{D}$ case) is the compliance contributed by the passives elements of the fingers (flexible fingertips, cables, etc.), $C_{q} \in \mathbb{R}^{m n \times m n}$ is the compliance contributed by the servoing of the joints and $J_{h} \in \mathbb{R}^{3 n \times m n}$ (resp. $\mathbb{R}^{2 n \times m n}$ for $2 \mathrm{D}$ case) is the hand Jacobian depending on the fingers' configuration $q$, with $m$ the number of joints per finger.

Let us introduce the transformation $T w_{w \rightarrow o b j}$ that transposes a wrench from the frame $w$ to the frame obj (equivalent to the adjoint transformation defined by Murray [4]), and the transformation $T p_{w \rightarrow o b j}$ that transposes a point's coordinates from the frame $w$ to the frame $o b j$, both being dependent on the configuration $X_{o}^{w}$ of the object in the world frame:

$$
\begin{aligned}
W_{d p \rightarrow o b j}^{o} & =T w_{w \rightarrow o b j}\left(X_{o}^{w}\right) W_{d p \rightarrow o b j}^{w} \\
\delta X_{o}^{o} & =T p_{w \rightarrow o b j}\left(X_{o}^{w}\right) \delta X_{o}^{w}
\end{aligned}
$$

Based on these definitions, we are now ready to expose the grasp stability analysis method.

\section{ANALYSIS OF GRASP STABILITY}

For a system in three dimensions, with $n$ contact points on the object, and $m$ constraints by contact point, a given set of contact points $X_{c}^{o}$ and joint stiffness $k_{p_{i j}}$ contributed by the servoing of the joints, the internal forces $f_{i}$, as well as configuration of the fingers $q_{i}, E_{d_{w_{o}} \max }$ is computed by solving the optimization problem presented in Algorithm 1. The maximal magnitude of the perturbation wrench in the given direction $d_{W_{e x t}}$ under which the system stays in the equilibrium region is also provided. The subscript $\bullet^{f b}$ is used to express quantities in the finger bases frame. The matrix $T p_{w \rightarrow f b}$ is the transformation used to express the coordinates of a point respectively from the world frame 
to the finger bases frames. The finger bases frames are the frames attached to the base of each finger, and each contact point is transposed to the frame attached to it's own finger. The variables $X_{c}^{o}, X_{c_{(k)}}^{w}$ and $X_{c_{(k)}}^{f b}$ are the concatenation of the coordinates of the contact points respectively in the object frame, world frame and finger bases frames. The function $i \mathrm{~km}$ is the inverse kinematic model. $\mu$ denotes the friction coefficient depending on the properties of the contact surfaces between the object and the fingertips.

At each step $\delta \alpha$ on the optimization, the state of the system is computed from the previous state using the function $N e w_{-} \operatorname{State}\left(\delta W_{e x t \rightarrow o b j}^{w}\right)$, then the equilibrium of the new state is verified using the frictions cone constraints of the grasp object.

Once $\alpha_{\max }$ is obtained, it is possible to get the corresponding $W_{e x t \rightarrow o b j}^{w}$ and $X_{o}$ and therefore the potential energy $E_{d_{w_{o}} \max }$.

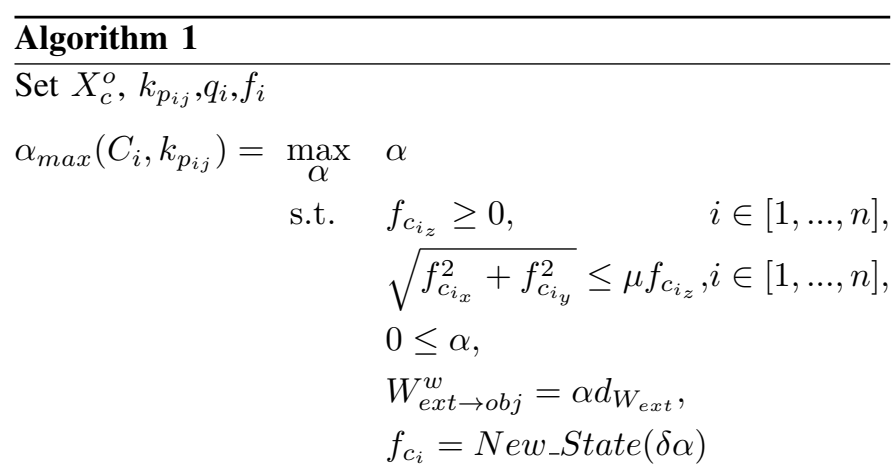

1: function NeW_State $\left(\delta W_{e x t \rightarrow o b j}^{w}\right)$

2: // Transposition of $\delta W_{e x t \rightarrow o b j}$ from $w$ to $o b j$ and calculation of the equivalent motion in $o b j$

3: $\quad \delta W_{e x t \rightarrow o b j_{(k)}}^{o} \leftarrow T w_{o b j \rightarrow w}\left(X_{o_{(k-1)}}\right) . \delta W_{e x t \rightarrow o b j}^{w}$

4: $\quad \delta X_{o_{(k)}}^{o} \leftarrow\left[G K_{c_{(k-1)}} G^{T}\right]^{-1} \delta W_{e x t \rightarrow o b j_{(k)}}^{o}$

5: // Calculation of the new $X_{o_{(k)}}$ and $W_{e x t \rightarrow o b j_{(k)}}^{w}$ in $w$

6: $\quad X_{o_{(k)}} \leftarrow X_{o_{(k-1)}}+T p_{o b j \rightarrow w}\left(X_{o_{(k)}}\right)^{T} \delta X_{o_{(k)}}^{o}$

7: $\quad W_{e x t \rightarrow o b j_{(k)}}^{w} \leftarrow W_{e x t \rightarrow o b j_{(k)}}^{w}+\delta W_{e x t \rightarrow o b j_{(k)}}^{w}$

8: // Calculation of the new hand's configuration

9: $\quad X_{c_{(k)}}^{w} \leftarrow T p_{o b j \rightarrow w}\left(X_{o_{(k)}}\right) X_{c}^{o}$

10: $\quad X_{c_{(k)}}^{f b} \leftarrow T p_{w \rightarrow f b} X_{c_{(k)}}^{w}$

11: $\quad q_{i(k)} \leftarrow i k m\left(X_{c_{(k)}}^{f b}\right)$

12: // New Stiffness matrix

13: $\quad K_{c_{(k)}}=\left(C_{s}+J_{h}\left(q_{i(k)}\right) C_{q}\left(k_{p_{i j}}\right) J_{h}\left(q_{i(k)}\right)^{T}\right)^{-1}$

14: // Calculation of the reaction forces and the stored energy induced by the motion

15: $\quad f_{r_{(k)}} \leftarrow f_{r_{(k-1)}}-K_{c_{(k-1)}} G^{T} \delta X_{o_{(k)}}^{o}$

16: $\quad E_{(k)} \leftarrow E_{(k-1)}+X_{o_{(k)}}^{o^{T}} G K_{c_{(k-1)}} G^{T} \delta X_{o_{(k)}}^{o}$

17: $\quad$ return $\left(f_{r_{(k)}}+f_{i}\right)$

18: end function
Hence a given $\alpha_{\max }$ corresponds to an energy $E_{d_{w_{o}} \max }$, a wrench $W_{e x t \rightarrow o b j}^{w}$ as well as a configuration $X_{o}$, that are respectively the maximum amount of energy, for a given direction of disturbance, the maximum intensity of the disturbance acceptable by the system before the cone constraints are violated and the new position where the grasp is at equilibrium under the said disturbance. These three quantities are linked among themselves and are dependent on the contacts points, the grasp stiffness and the internal forces. As such it is possible to optimize $E_{d_{w_{o}} \max }$ or $W_{e x t \rightarrow o b j}^{w}$ using these three variables.

As the consequences in terms of disturbance wrenches can be more direct to interpret than the energy, the rest of the article will focused on this quantity.

\section{CASE STUDY}

This section illustrates our approach based on an example of a planar pick and place task with a hand. This example will show how the tuning of the stiffness and the contact point position in the system can improve its robustness to external disturbance.

\section{A. Considered System}

The following ideal planar 2D example relates to a grasp with two fingers and two contact points and Coulomb friction constraints (Fig. 2), $f_{c}=\left[\begin{array}{llll}f_{c 1_{x}} & f_{c 1_{y}} & f_{c 2_{x}} & f_{c 2_{y}}\end{array}\right]^{T} \in \mathbb{R}^{4}$ and $W_{e x t \rightarrow o b j}^{w}=\left[\begin{array}{lll}W_{x} & W_{y} & W_{\theta}\end{array}\right]^{T} \in \mathbb{R}^{3}$. Let us denote $m=1 \mathrm{~kg}$ the mass of the grasped object.

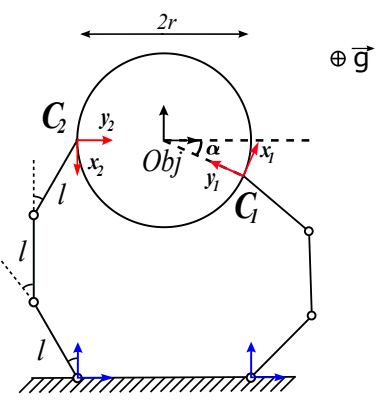

Fig. 2: Studied system

with the radius of the object fixed to $2 r=0.2 \mathrm{~m}$, the length of the segments of the finger fixed to $l=0.1 \mathrm{~m}$ and the friction coefficient fixed to $\mu=1$.

Moreover, let us assume that the relationship between the deformation of the fingertips and the reaction force is expressed by a linear spring model, and that the servoing of the joints of the hand is a proportional servoing. The matrix $C_{s}$ and $C_{q}$ can therefore be defined as:

$$
\begin{aligned}
& C_{q}=\left[\begin{array}{cc}
C_{q_{1}} & \mathbb{O} \\
\mathbb{O} & C_{q_{2}}
\end{array}\right] \\
& C_{s}=\left[\begin{array}{cc}
C_{s_{1}} & \mathbb{O} \\
\mathbb{O} & C_{s_{2}}
\end{array}\right]
\end{aligned}
$$


with $C_{q_{i}}=\left[\begin{array}{ccc}\frac{1}{k p_{i 1}} & 0 & 0 \\ 0 & \frac{1}{k p_{i 2}} & 0 \\ 0 & 0 & \frac{1}{k p_{i 3}}\end{array}\right], C_{s_{i}}=\left[\begin{array}{cc}C_{s_{i} x} & 0 \\ 0 & C_{s_{i} y}\end{array}\right]$ for $i \in\{1,2\}, k p_{i 1}, k p_{i 2}, k p_{i 3}$ being the proportional gains of respectively the first, second and last joint of the $i$-th finger starting from the base, and $C_{s_{i} x}$ and $C_{s_{i} y}$ being the tangential and normal compliance of the $i$-th finger's fingertip.

\section{B. Pick \& Place example task}

For this example, we define a trajectory along which the system hand-object is supposed to move. This trajectory is defined by four way-points to be exactly reach at zero velocity and three point-to-point paths in between.

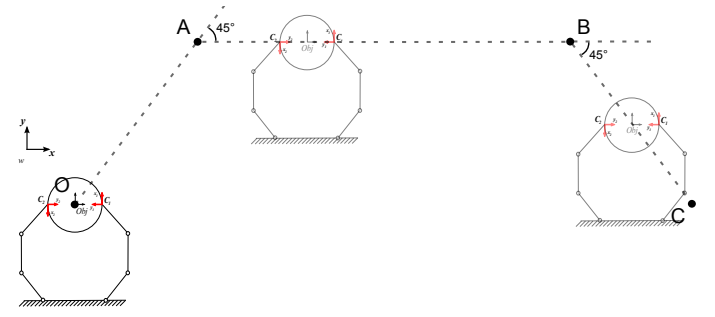

Fig. 3: Pick \& place trajectory

During the trajectory, the object is subject to several directions of acceleration expressed in frame $w$ :

- From $O$ to $A$, an acceleration in the direction $\left[\begin{array}{lll}-\sqrt{2} / 2 & -\sqrt{2} / 2 & 0\end{array}\right]^{T}$ then $\left[\begin{array}{lll}\sqrt{2} / 2 & \sqrt{2} / 2 & 0\end{array}\right]^{T}$

- From $A$ to $B$, an acceleration in the direction $\left[\begin{array}{ccc}-1 & 0 & 0\end{array}\right]^{T}$ then $\left[\begin{array}{ccc}1 & 0 & 0\end{array}\right]^{T}$

- From $B$ to $C$, an acceleration in the direction $\left[\begin{array}{lll}-\sqrt{2} / 2 & \sqrt{2} / 2 & 0\end{array}\right]^{T}$ then $\left[\begin{array}{lll}\sqrt{2} / 2 & -\sqrt{2} / 2 & 0\end{array}\right]^{T}$

From the second Newton's Law, it can be deduced the external disturbance directions listed in (22):

$$
d_{w_{\text {ext }}} \in\left\{\left[\begin{array}{c}
-\frac{\sqrt{2}}{2} \\
-\frac{\sqrt{2}}{2} \\
0
\end{array}\right],\left[\begin{array}{c}
\frac{\sqrt{2}}{2} \\
\frac{\sqrt{2}}{2} \\
0
\end{array}\right],\left[\begin{array}{c}
-1 \\
0 \\
0
\end{array}\right],\left[\begin{array}{l}
1 \\
0 \\
0
\end{array}\right],\left[\begin{array}{c}
\frac{-\sqrt{2}}{2} \\
\frac{\sqrt{2}}{2} \\
0
\end{array}\right],\left[\begin{array}{c}
\frac{\sqrt{2}}{2} \\
-\frac{\sqrt{2}}{2} \\
0
\end{array}\right]\right\}
$$

\section{Analysis for a fixed grasp}

In a case where all the parameters of the grasp are fixed, it is possible using the method explained in the algorithm 1 to get the maximal acceleration intensity that the system can withstand for each section of the trajectory.

Let us set the internal force to $f_{i}=5 \times\left[\begin{array}{llll}0 & 1 & 0 & 1\end{array}\right]^{T} \mathrm{~N}$, the same proportional coefficients for all joints $k_{p_{i j}}=$ $5 \mathrm{~N} / \mathrm{rad}(i \in\{1,2\}, j \in\{1,2,3\})$ and the initial joint configuration for each finger $q_{1}=\left[\begin{array}{lll}-\pi / 6 & \pi / 6 & \pi / 6\end{array}\right]^{T}$ and $q_{2}=\left[\begin{array}{lll}\pi / 6 & -\pi / 6 & -\pi / 6\end{array}\right]^{T}$.

The table I gathers the results for the quantities $\Delta E_{\min }$ and $W_{e x t \rightarrow o b j}^{w}$ for each direction of perturbation undergone by the object along the trajectory. The table II is the equivalent acceleration for each section of the trajectory. First, it can be seen that the system is symmetrical with respect to the world $y$-axis. Second, although the size of the maximal accelerations is the same, their values is dependent on the direction. Thus, selecting the same acceleration profile for all the sections of the trajectory would not be the best option.

TABLE I: Results for the various $d_{w_{e x t}}$

\begin{tabular}{|c|c|c|}
\hline Disturbance directions & $\Delta E_{\min }(m \mathrm{~J})$ & $W_{e x t \rightarrow o b j}^{w}$ \\
\hline$\left[\begin{array}{lll}-\frac{\sqrt{2}}{2} & -\frac{\sqrt{2}}{2} & 0\end{array}\right]^{T}$ & 0.706 & {$\left[\begin{array}{lll}-0.248 & -0.248 & 0\end{array}\right]^{T}$} \\
\hline$\left[\begin{array}{lll}\frac{\sqrt{2}}{2} & \frac{\sqrt{2}}{2} & 0\end{array}\right]^{T}$ & 0.429 & {$\left[\begin{array}{lll}0.339 & 0.339 & 0\end{array}\right]^{T}$} \\
\hline$\left[\begin{array}{lll}-1 & 0 & 0\end{array}\right]^{T}$ & 0.761 & {$[-0.280 \quad 0 \quad 0]^{T}$} \\
\hline$\left[\begin{array}{lll}1 & 0 & 0\end{array}\right]^{T}$ & 0.761 & {$\left[\begin{array}{lll}0.280 & 0 & 0\end{array}\right]^{T}$} \\
\hline$\left[\begin{array}{lll}-\frac{\sqrt{2}}{2} & \frac{\sqrt{2}}{2} & 0\end{array}\right]^{T}$ & 0.429 & {$\left[\begin{array}{lll}-0339 & 0.339 & 0\end{array}\right]^{T}$} \\
\hline$\left[\begin{array}{lll}\frac{\sqrt{2}}{2} & -\frac{\sqrt{2}}{2} & 0\end{array}\right]^{T}$ & 0.706 & {$\left[\begin{array}{lll}0.248 & -0.248 & 0\end{array}\right]^{T}$} \\
\hline
\end{tabular}

TABLE II: Maximal acceleration norms for each section of the trajectory

\begin{tabular}{lc}
\hline Trajectory Segment & $\max (\|\dot{v}(t)\|)\left(\mathrm{m} / \mathrm{s}^{2}\right)$ \\
\hline$[O A]:$ beginning & 0.480 \\
{$[O A]:$ end } & 0.350 \\
{$[A B]:$ beginning } & 0.280 \\
{$[A B]:$ end } & 0.280 \\
{$[B C]:$ beginning } & 0.350 \\
{$[B C]:$ end } & 0.480 \\
\hline
\end{tabular}

\section{Grasp selection}

Conversely to the previous analysis, given a fixed set of disturbance wrenches to resist, the Algorithm 1 can be used to select a suitable grasp.

Let us assume the same pick-and-place task described in Fig. 3, and let us fix the maximal acceleration to 0.7 meter per second. Such acceleration implies that the system should be able to resist at least a disturbance intensity $\left|W_{e x t \rightarrow o b j}^{w}\right|_{\min }=0.7 \mathrm{~N}$ for every directions listed in (22).

The system has several degrees of freedom to set the correct parameters (contact points, joint stiffness, internal forces), so it is possible to get the regions of parameters for which the following constraint is verified:

$$
\left|W_{e x t \rightarrow o b j}^{w}\right| \geq\left|W_{e x t \rightarrow o b j}^{w}\right|_{\text {min }}
$$

For example, if we take a look at the evolution of $\left|W_{e x t \rightarrow o b j}^{w}\right|$ depending on the position of the $X_{c_{1}}$ contact point of the right fingertip on the object and an homogeneous proportional coefficient $k_{p_{i}}$ on all the joints, we can get the couples $\left(k_{p_{i j}}, X_{c_{1}}\right)$ such as the constraint (23) is valid. The Figure 4 represents the sets $\mathbb{S}_{d_{k}}$ of admissible couple $\left(k_{p_{i j}}, X_{c_{1}}\right)$ for each direction $d_{k}$ listed in (22). The set $\mathbb{S}^{*}=\mathbb{S}_{d_{1}} \cap \mathbb{S}_{d_{2}} \cdots \cap \mathbb{S}_{d_{6}}$ is the set of admissible couple $\left(k_{p_{i j}}, X_{c_{1}}\right)$ for the whole task.

The contact point $X_{c_{1}}$ is parameterized by a radial angle $\alpha_{X_{c_{1}}} \in[-1,1]$ between the horizontal line going through the center of the object and the straight line going through the contact point and the center of the object (Fig. 3), and $k_{p_{i j}} \in[0.02,2]$. 


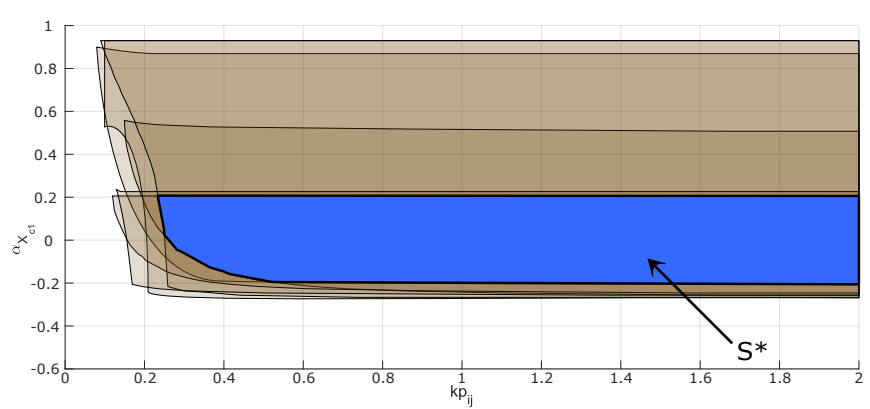

Fig. 4: Sets of pairs $\left(k_{p_{i j}}, X_{c_{1}}\right)$ leading to a stable grasp under a disturbance wrench's intensity of $0.7 \mathrm{~N}$

The figures 5 and 6 show two different grasps. On both figures, the black and gray arrows stand for the maximals forces that can be withstood by the hand in their respective directions, the black ones being the forces considered for the task. The dotted circle stands for the minimal of intensity set to 0.7 Newtons, and the $P_{\text {move }}$ area stand for the region where the object center of mass can move without slippage of the finger, i.e the equilibrium region.

The first grasp $\left(k_{p_{i j}}=0.3, \alpha_{X_{c_{1}}}=0\right)$ is present in the valid set, and it can be seen by the fact that all the intensities of the forces listed in (22) are outside the minimal boundary (i.e. the black ones). As such this grasp is able to resist at least a force equal to 0.7 Newtons in intensity. (a) Maximum tolerated wrenches

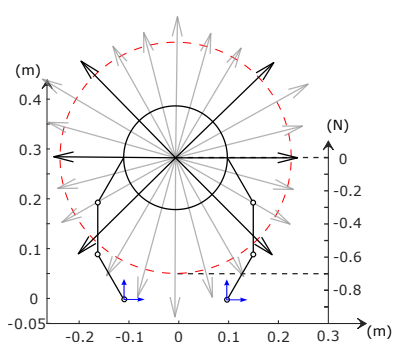

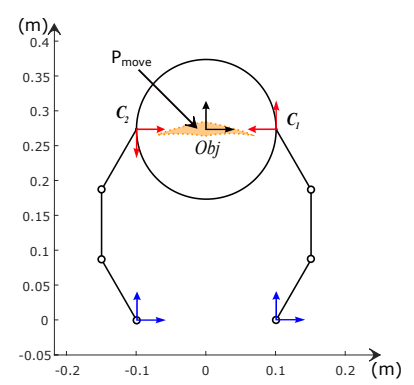

(b) Equilibrium region $P_{\text {move }}$
Fig. 5: Case where $\left(k_{p_{i j}}=0.3, \alpha_{X_{c_{1}}}=0\right) \in \mathbb{S}^{*}$ (Arrows denote the maximal tolerated wrench for each direction)

The second grasp $\left(k_{p_{i j}}=0.3, \alpha_{X_{c_{1}}}=-\frac{\pi}{8}\right)$ is not in the set $\mathbb{S}^{*}$ and in can be seen in the figure 6. Although four out of six directions are out of the circle, this grasp does not ensure the stability for every directions. Thus it is not suitable for this task, but in some directions this grasp is better than the first one, thus it will be more suitable for a task that use these kind of directions instead.

Although it's not a criteria in this study case, we can see that the equilibrium region of the second grasp is far smaller than the first grasp. As such the second grasp could also be more suitable for a task that is constrained in displacement.

\section{CONCLUSIONS}

This paper introduced a stability analysis taking explicitly into account the whole geometry of the hand as well as the compliance due to the mechanical design and the control gains in the fingertips and the joints. This analysis is based on

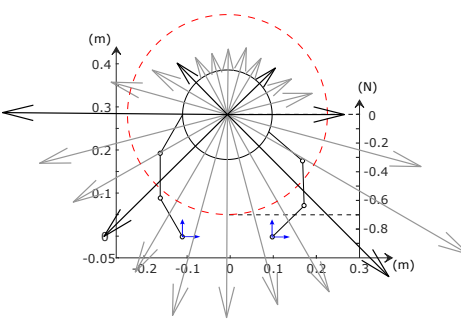

(a) Maximum tolerated wrenches

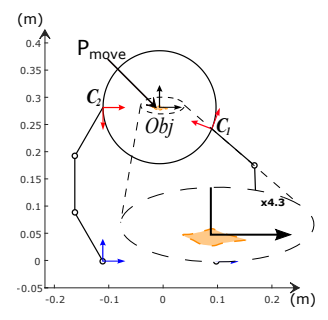

(b) Equilibrium region $P_{\text {move }}$
Fig. 6: Case where $\left(k_{p_{i j}}=0.3, \alpha_{X_{c_{1}}}=-\frac{\pi}{8}\right) \notin \mathbb{S}^{*}$ (Arrows denote the maximal tolerated wrench for each direction)

the greatest disturbance wrench that the grasp can withstand for a given direction as well as the energy and displacement induced by this wrench. This approach was exemplified on a simple 2D case where only the maximal wrench criterion was used to select a grasp robust to external disturbances (other criteria could include the energy or the displacement).

The stability analysis has been illustrated with the ideal planar pick-and-place task in two ways, the first one to estimate for a given grasp, the maximum accelerations that the hand and object can withstand, and a second one to evaluate the set of grasp parameters that guarantee the stability along the whole trajectory given a minimal acceleration to which the grasp is supposed to resist.

Although this stability analysis is not global, it still gives relevant informations for the selection of a grasp suitable for a definite task, or can also help to tune the task's trajectory to restrain the related disturbances in a scale bearable by the system. Future work will be dedicated to the extension of the proposed method to the $3 \mathrm{D}$ case as well as the enveloping grasps. Adaptive command will also be considered to optimize the control gains with respect to the trajectory.

\section{REFERENCES}

[1] M. Grossard, M. Martin Amezaga, and G. Felipe, "Control-oriented design and robust decentralized control of the cea dexterous robot hand," vol. 20, 2014, pp. 1809-1821.

[2] H. Bruyninckx, S. Demeyt, and V. Kumar, "Generalized stability of compliant grasps," in Proc. IEEE International Conference on Robotics and Automation (ICRA'98), Lxuven, Belgium, May 1998, pp. 23962402.

[3] V.-D. Nguyen, "The synthesis of stable grasps in the plane," in Proc. IEEE International Conference on Robotics and Automation (ICRA'86), San Francisco, CA, USA, USA, Apr. 1986, pp. 884-889.

[4] R. M. Murray, Z. Li, and S. S. Sastry, A Mathematical Introduction to Robotic Manipulation. Boca Raton, Florida, United States: CRC Press, 1994.

[5] V.-D. Nguyen, "Constructing force-closure grasps," The International Journal of Robotics Research, vol. 7, no. 3, pp. 3-16, 1988.

[6] W. S. Howard and V. Kumar, "On the stability of grasped objects," in IEEE Transactions on Robotics and Automation. IEEE, 1996, vol. 12, no. 6, pp. 904-917.

[7] M. R. Cutkosky and I. Kao, "Computing and controlling the compliance of a robotic hand," IEEE J. Robot. Automat., vol. 5, pp. 151-165, Apr. 1989.

[8] T. Tsuji, K. Baba, et al., "Grasp stability analysis for elastic fingertips by using potential energy," in Proc. IEEE International Symposium on System Integration, Tokyo, Japan, Dec. 2014, pp. 453-458.

[9] D. Prattichizzo, M. Malvezzi, M. Gabiccini, and A. Bicchi, "On the manipulability ellipsoids of underactuated robotic hands with compliance," Rob. Auton. Syst., vol. 60, no. 3, pp. 337-346, 2012. 